\title{
The Transition to Aperiodic Behavior in Turbulent Systems
}

\author{
Mitchell J. Feigenbaum \\ University of California, Los Alamos Scientific Laboratory (T-DOT MS 452), P.O. Box 1663, Los \\ Alamos, NM 87545, USA
}

\begin{abstract}
Some systems achieve aperiodic temporal behavior through the production of successive half subharmonics. A recursive method is presented here that allows the explicit computation of this aperiodic behavior from the initial subharmonics. The results have a character universal over specific systems, so that all such transitions are characterized by noise of a universal internal similarity.
\end{abstract}

\section{Introduction}

A variety of numerical experiments [1-6] on systems of differential equations have demonstrated that a possible route to chaotic or turbulent behavior is a cascade of successive half harmonics of a basic mode with turbulence commencing after an infinite halving has produced non-periodic behavior. Moreover, a recent experiment [7] on Rayleigh Bénard convection has also exhibited these half harmonics as the behavior determining the onset of turbulence in the fluid. In this paper we draw upon some mathematics intimately connected with period doubling, and determine the time fluctuation spectrum of such a system at the onset of turbulence [8]. This spectrum proves to be of a universal construction, so that no specific formulas for the differential system are ever encountered. On the other hand, the theory presented is asymptotic and recursive, so that it requires as input the specific spectrum after several stages of period doubling. We are not concerned with this aspect here, taking this input for granted after which the entirety of the behavior through the transition is computed.

The paper is divided into three main parts. The first assumes the theory later to be exposited in order to present the new results with the least dedication required of the reader. The principal results are the formulae (10) and (17) which determine the subharmonic spectrum recursively. The second section reviews the universality theory for one-dimensional maps, and constructs the basic scaling function required in the first part. Finally, the last section consists of an argument establishing the relevance of one-dimensional maps to the original system of differential equations, resting upon very recent work of Collet et al. [9]. The correct formula to which (10) is a rough approximation is also determined. 


\section{Subharmonic Trajectory Scaling}

We are considering a system of differential equations

$$
\dot{x}_{i}=f_{i}\left(x_{1}, \ldots, x_{N}, \lambda\right) i=1, \ldots, N
$$

for which we know that at $\lambda_{n}$ each $x_{i}(t)$ is periodic with period $T_{n}=2^{n} T_{0}$ and $\lambda_{n} \rightarrow \lambda_{\infty}<\infty$. (For each $T_{n}$ there is a range of suitable $\lambda$ 's : $\lambda_{n}$ is chosen for each $n$ to produce identical stabilities.) As $n \rightarrow \infty, x_{i}(t)$ is becoming aperiodic: it possesses a fundamental at $\omega_{n}=\omega_{0} / 2^{n}$ with equally spaced harmonics, with $2^{n}$ harmonics up to $\omega_{0}$, so that as $n \rightarrow \infty$, the spectrum becomes continuous. We are, in particular, interested in computing this part of the spectrum - the subharmonics of $\omega_{0}$ - that determines the noise (or fluctuations).

Consider the system at $\lambda_{n}: x_{i}\left(t+T_{n}\right)=x_{i}(t)$, and the motion can be divided into $2^{n}$ roughly similar "cycles" of duration $T_{0}$. At $\lambda_{n+1}, x_{i}\left(t+T_{n}\right)$ no longer equals $x_{i}(t)$, but "almost" does, another $T_{n}$ "cycles" required until $x_{i}$ again equals $x_{i}(t)$. Accordingly, we focus attention on

$$
\psi^{(n)}(t) \equiv x^{(n)}(t)-x^{(n)}\left(t+T_{n-1}\right)
$$

which along the entire trajectory measures $x$ 's failure to duplicate itself after one half of its true period, $T_{n}$. Observe, by periodicity, that

$$
\psi^{(n)}\left(t+T_{n}\right)=\psi^{(n)}(t)
$$

and

$$
\psi^{(n)}\left(t+T_{n-1}\right)=-\psi^{(n)}(t)
$$

Now imagine that the "way" in which $\psi^{(n)}$ fails to vanish is the same way in which $\psi^{(n+1)}$ will fail to vanish. More precisely, assume that

$$
\psi^{(n+1)}(t) \cong \sigma\left(t / T_{n+1}\right) \psi^{(n)}(t)
$$

so that $\psi^{(n+1)}$ is built of two displaced copies of $\psi^{(n)}(t)$ suitably scaled. By (3) it follows that

$$
\begin{gathered}
\sigma(x+1)=\sigma(x) \\
\sigma(x+1 / 2)=-\sigma(x) .
\end{gathered}
$$

Also, by (4)

$$
r_{n+1}(2 t) \equiv \frac{\psi^{(n+1)}(2 t)}{\psi^{(n)}(2 t)} \cong \sigma\left(2 t / T_{n+1}\right)=\sigma\left(t / T_{n}\right) \cong \frac{\psi^{(n)}(t)}{\psi^{(n-1)}(t)}=r_{n}(t)
$$

so that with each $r_{n}$ plotted against a scaled time, $x$, (for which $T_{n}=1$ ), the curves $r_{n}(t)$ should be identical and equal to $\sigma(x)$. Figure 1 depicts the degree to which this is true for $n=4,5$ in the case of Duffing's equation, where $\lambda_{n}$ has been chosen to determine that $2^{n}$-cycle most quickly converged to from initial conditions within the basin of this cycle. In fact there is a large class of systems (1) for which Eq. (4) is correct. So, assuming that (4) is correct (for large n), let us see what form of fluctuation spectrum is implied. 


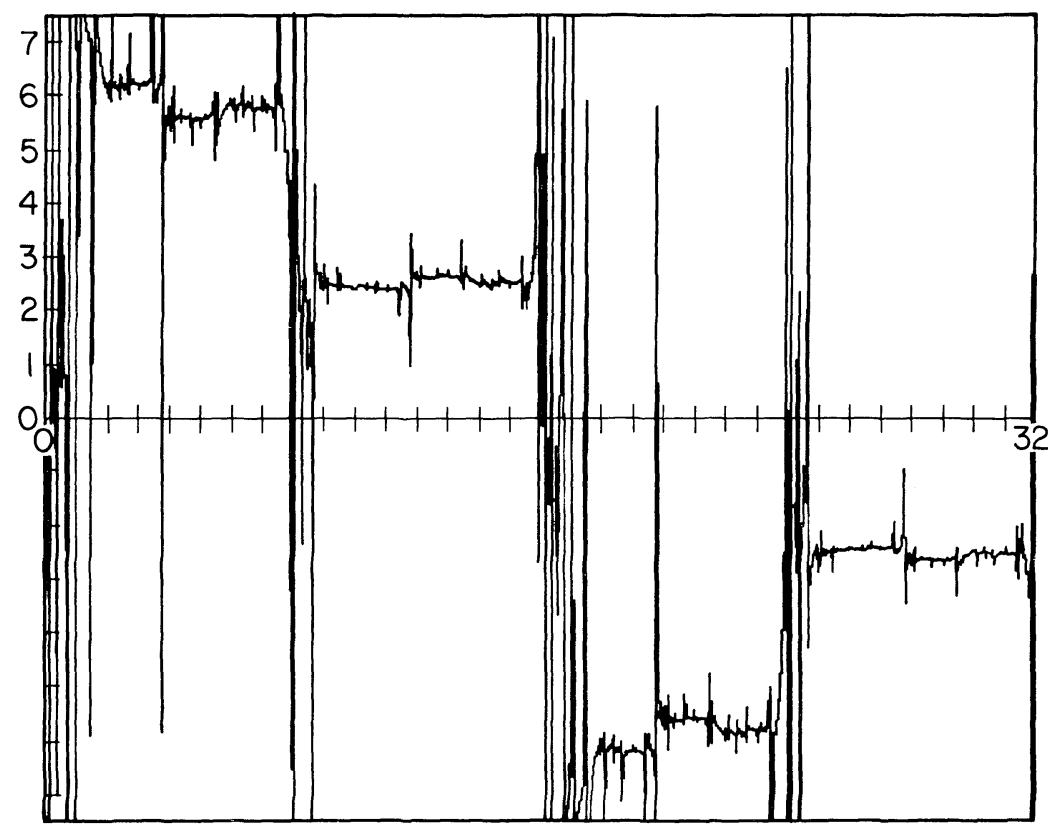

a

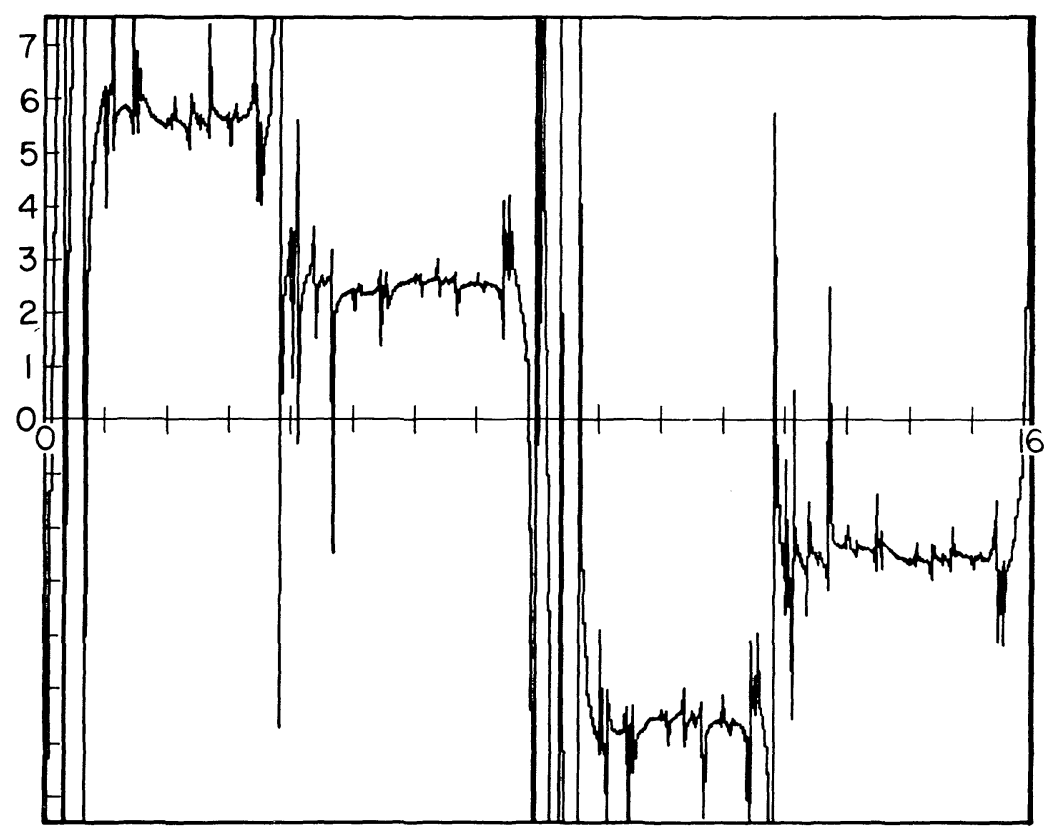

b

Fig. 1. a $r_{5}^{-1}(t) ; \mathbf{b} r_{4}^{-1}(t) ; r_{n}^{-1}$ as obtained by the direct numerical division of solutions to Duffing's equation. The divergences represent slight mismatches of zeroes, and are, for smooth integrals of (4), irrelevant 
First of all, the even and odd Fourier components of $x^{(n)}$ are fundamentally of different characters. This is so simply because at $\lambda_{n+1}$, the odd harmonics of the fundamental, $\omega_{0} / 2^{n+1}$, are all absent in the spectrum at $\lambda_{n}$, since at $\lambda_{n}$ the fundamental is $\omega_{0} / 2^{n}=2 \omega_{0} / 2^{n+1}$. Thus at $\lambda_{n+1}$, a set of new components beyond those of $\lambda_{n}$ are introduced, while those present at $\lambda_{n}$ are located coincidentally with the even harmonics at $\lambda_{n+1}$. In fact, to a first approximation, the even components at $\lambda_{n+1}$ are just all the old components at $\lambda_{n}$, so the main task in determining the spectrum at $\lambda_{n+1}$ is the computation of the new components. Let us now formally render these verbal remarks, in order to see what we must compute. By definition, the $k$ th Fourier component of $x^{(n)}(t)$ is

$$
x_{k}^{(n)} \equiv \int_{0}^{T^{n}} \frac{d t}{T_{n}} x^{(n)}(t) e^{-2 \pi i k t / T_{n}} .
$$

For our purposes, we manipulate (7) by splitting the integral into two halves, and shifting the upper half, producing,

$$
x_{k}^{(n)}=\int_{0}^{T_{n-1}} \frac{d t}{2 T_{n-1}}\left[x^{(n)}(t)+(-1)^{k} x^{(n)}\left(t+T_{n-1}\right)\right] e^{-\pi i k t / T_{n-1}} .
$$

Consider first the even harmonics of $x^{(n+1)}$. By (8),

$$
x_{2 k}^{(n+1)}=\int_{0}^{T_{n}} \frac{d t}{2 T_{n}}\left[x^{(n+1)}(t)+x^{(n+1)}\left(t+T_{n}\right)\right] e^{-2 \pi i k t / T_{n}} .
$$

However, after $T_{n}, x^{(n+1)}$ has almost repeated itself ( $\sigma$ is small), so that to first approximation,

$$
x^{(n+1)}(t) \cong x^{(n+1)}\left(t+T_{n}\right) \cong x^{(n)}(t)
$$

and by (9),

$$
x_{2 k}^{(n+1)} \cong \int_{0}^{T_{n}} \frac{d t}{T_{n}} x^{(n)}(t) e^{-2 \pi i k / T_{n}}=x_{k}^{(n)} .
$$

However, the fundamental of $x^{(n+1)}$ is $\omega_{0} / 2^{n+1}$, so that its $2 k^{\text {th }}$ harmonic is at $\omega=k \omega_{0} / 2^{n}$ which is the $k t h$ harmonic of the fundamental $\omega_{0} / 2^{n}$ of $x^{(n)}$. Thus, as verbally stated, to first approximation, the even harmonics at $\lambda_{n+1}$ are just the spectrum at $\lambda_{n}$.

Accordingly, we now turn to the serious computation, that for the odd harmonics. By (8),

$$
x_{2 k+1}^{(n+1)}=\int_{0}^{T_{n}} \frac{d t}{2 T_{n}}\left[x^{(n+1)}(t)-x^{(n+1)}\left(t+T_{n}\right)\right] e^{-\pi i(2 k+1) t / T_{n}} .
$$

The integrand of $(11)$ is $\psi^{(n+1)}(t)$, so that by (4),

$$
x_{2 k+1}^{(n+1)} \cong \int_{0}^{T_{n}} \frac{d t}{2 T_{n}} \sigma\left(t / 2 T_{n}\right)\left[x^{(n)}(t)-x^{(n)}\left(t+T_{n-1}\right)\right] e^{-\pi i(2 k+1) t / T_{n}} .
$$

Using the inverse of (7),

$$
x^{(n)}(t)=\sum_{k} x_{k}^{(n)} e^{2 \pi i k t / T_{n}}
$$


we have

$$
x^{(n)}(t)-x^{(n)}\left(t+T_{n-1}\right)=2 \sum_{k} x_{2 k+1}^{(n)} e^{2 \pi i\left(2 k^{\prime}+1\right) t / T_{n}} .
$$

Substituting (13) into (12),

$$
x_{2 k+1}^{(n+1)} \cong \sum_{k} x_{2 k^{\prime}+1}^{(n)} \int_{0}^{T_{n}} \frac{d t}{T_{n}} \sigma\left(t / 2 T_{n}\right) e^{2 \pi i\left[\left(2 k^{\prime}+1\right)-1 / 2(2 k+1)\right] t / T_{n}},
$$

which, through the substitution $\xi=t / T_{n}$, becomes

$$
x_{2 k+1}^{(n+1)} \cong \sum_{k^{\prime}} x_{2 k^{\prime}+1}^{(n)} \int_{0}^{1} d \xi \sigma(\xi / 2) e^{2 \pi i \xi\left[\left(2 k^{\prime}+1\right)-1 / 2(2 k+1)\right]} .
$$

Thus, the scaling law (4) allows the computation of the spectrum of $x^{(n+r)}(t)$ for all $r \geqq 1$, given the spectrum of $x^{(n)}(t)$. Equation (14) has two significant properties. First, only the odd components of $x^{(n)}$ are required to determine the new (and odd) components of $x^{(n+1)}$. This is important because the "cycle" frequency, $\omega_{0}$, is the $2^{n}$ harmonic of $\omega_{n}$ and so even. That is, the basic "cycle"s spectrum is decoupled from (14), so that the noise spectrum introduced at each $n$ is roughly independent of the "coherent" spectrum of the basic "cycle". Also significant is that the recursion (14) is independent of $n$ (i.e. "autonomous").

It should be pointed out that if (4) is asymptotically exact as $n \rightarrow \infty$, then (14) is similarly exact. Formula (10) however is genuinely approximate, and we defer until later the correct formula, since an extra ingredient is required.

By Fig. 1, $\sigma$ is marked by discontinuities (as $n \rightarrow \infty$ ), roughly constant at one value for $0<x<1 / 4$ and constant at another for $1 / 4<x<1 / 2$. In a next approximation, each of these quarters is decomposable into two halves, and so forth. Thus, $\sigma$ is representable as

$$
\sigma(x)=\sum \sigma_{i} \theta\left(x_{i+1}-x\right) \theta\left(x-x_{i}\right) .
$$

Let us use (15) to compute the transform integral of (14):

$$
I([]) \equiv \int_{0}^{1} d \xi \sigma(\xi / 2) e^{2 \pi i \xi[]}=\left.\frac{1}{2 \pi i[]} \sigma(\xi / 2) e^{2 \pi i \xi[]}\right|_{0} ^{1}-\frac{1}{4 \pi i[]} \int_{0}^{1} d \xi \sigma^{\prime}(\xi / 2) e^{2 \pi i \xi[]} .
$$

Since $e^{2 \pi i\left[\left(2 k^{\prime}+1\right)-1 / 2(2 k+1)\right]}=-1$,

$$
I([])=-\frac{1}{2 \pi i[]}\left\{\left(\sigma\left(1 / 2^{-}\right)+\sigma\left(0^{+}\right)\right)+1 / 2 \int_{0}^{1} d \xi \sigma^{\prime}(\xi / 2) e^{2 \pi i \xi[]}\right\} .
$$

By (15),

$$
\sigma^{\prime}(x)=\sum \sigma_{i}\left(\delta\left(x-x_{i}\right)-\delta\left(x-x_{i+1}\right)\right)=\sum\left(\sigma_{i}-\sigma_{i-1}\right) \delta\left(x-x_{i}\right) \equiv \sum d_{i} \delta\left(x-x_{i}\right)
$$

so that

$$
1 / 2 \int_{0}^{1} d \xi \sigma^{\prime}(\xi / 2) e^{2 \pi i \xi[]}=\sum_{i} d_{i} \int_{0}^{1} \frac{d \xi}{2} \delta\left(\xi / 2-x_{i}\right) e^{2 \pi i \xi[]}=\sum_{i}\left(d_{i}\right) e^{4 \pi i x_{i}[]} .
$$


Accordingly, (14) becomes

$$
\begin{aligned}
x_{2 k+1}^{(n+1)} \cong & -\frac{1}{2 \pi i} \sum_{k} x_{2 k^{\prime}+1}^{(n)} \frac{1}{2 k^{\prime}+1-1 / 2(2 k+1)} \\
& \cdot\left\{\left(\sigma\left(1 / 2^{-}\right)+\sigma\left(0^{+}\right)\right)+\sum_{i} d_{i} e^{4 \pi i x_{i}\left[\left(2 k^{\prime}+1\right)-1 / 2(2 k+1)\right]}\right\} .
\end{aligned}
$$

From (16) follow various approximations by including only those discontinuities $d_{i}$ in excess of a certain amount. As a first approximation, we include only the largest discontinuity at $x=1 / 4$, for which the exponential factor is

$$
e^{\pi i\left[\left(2 k^{\prime}+1\right)-1 / 2(2 k+1)\right]}=i(-1)^{k},
$$

so that (16) becomes

$$
\begin{aligned}
x_{2 k+1}^{(n+1)} \cong & -\frac{1}{2 \pi i}\left[\left(\sigma\left(1 / 2^{-}\right)+\sigma\left(0^{+}\right)\right)+i(-1)^{k}\left(\sigma\left(1 / 4^{+}\right)-\sigma\left(1 / 4^{-}\right)\right)\right] \\
& \cdot \sum_{k^{\prime}} \frac{x_{2 k^{\prime}+1}^{(n)}}{2 k^{\prime}+1-1 / 2(2 k+1)}
\end{aligned}
$$

[To gauge the accuracy of (17), given the $n=4$ spectrum for Duffing's equation, the $n=5$ spectrum is determined to within $5 \%$ of the correct values for both amplitude and phase, with the logarithm of amplitudes determined to about $0.5 \%$.] As $T_{n} \rightarrow \infty$ (7) determines $x_{k}$ analytically continued in $k$ into the lower half plane, so that

$$
-\frac{P}{\pi i} \int \frac{d k^{\prime}}{k^{\prime}-k} x\left(k^{\prime}\right)=x(k) .
$$

An integral approximation to the sum of (17) immediately produces

$$
\left|x_{k}^{(n+1)}\right| \cong \frac{1}{4} \sqrt{\left(\sigma\left(1 / 2^{-}\right)+\sigma\left(0^{+}\right)\right)^{2}+\left(\sigma\left(1 / 4^{+}\right)-\sigma\left(1 / 4^{-}\right)\right)^{2}}\left|x^{(n)} k / 2\right| \equiv \mu\left|x^{(n)} k / 2\right| .
$$

The meaning of (18) is: smoothly interpolate the odd components of $x^{(n)}$ and rescale by $\mu$; the odd components of $x^{(n+1)}$ are "in the mean" the values of this new curve at the appropriate (odd) frequencies. Reducing by another factor of $\mu$ determines the $(n+2)$ spectrum and so forth ad infinitum. Accordingly, the fluctuation spectrum (approximately) has a simple self-similar character.

Thus, Fig. 1 completely determines the fluctuations spectrum for Duffing's equation as $n \rightarrow \infty$. What is remarkable though, is that Fig. 1 [i.e. $\sigma(x)$ ] is in fact universal over all systems (1) possessing order $2^{n}$ subharmonic production sequentially as $\lambda_{n} \rightarrow \lambda_{\infty}$ ! In the next section, we partially prove this result and compute $\sigma(x)$ in a universal format. Anticipating this computation, let us record here the quantities of (17) and (18) that will follow.

$$
\begin{aligned}
\sigma\left(1 / 2^{-}\right) & =0.3995 \quad \sigma\left(0^{+}\right)=\left[\sigma\left(1 / 2^{-}\right)\right]^{2}=0.1596 \\
\sigma\left(1 / 4^{+}\right) & =0.4191 \quad \sigma\left(1 / 4^{-}\right)=0.1752 \\
\mu & =0.1525 \\
-10 \log _{10} \mu & =8.17 \mathrm{db} .
\end{aligned}
$$


For log-amplitudes, (18) simply means that the new components at any level $n$ define roughly the same interpolation as the previous level's new components, but shifted $8.2 \mathrm{db}$ downwards. We now proceed with the theoretical notions and the computation of $\sigma(x)$.

\section{Universality Theory}

An elementary class of systems exhibiting successive period doublings has been known to exist for some time $[10,11]$. These are one-dimensional discrete systems : a one parameter family of (non-invertible) maps on an interval of the real line. For fixed parameter $\lambda$, a dynamics is determined by successive iteration of the map,

$$
x_{n+1}=f\left(\lambda, x_{n}\right) .
$$

$f$ has the crucial property of mapping an interval several-to-one onto itself, accomplished by $f$ 's attaining an extremum within the interval. For a large class of such $f$ 's there exists a monotone sequence of parameter values $\lambda_{n}$ such that at $\lambda_{n}$ a maximally stable $2^{n}$-cycle exists (or more generally, for any $r$, an $r \cdot 2^{n}$ cycle for appropriate $\lambda_{n}$ ).

Several years ago this author discovered that beyond this universal doubling property, the maps of this class possess a host of universal metric properties [1215]. More precisely, with $\hat{x}$ the location of the extremum of $f$, all $f$ 's with

$$
|f(x)-f(\hat{x})| \propto|x-\hat{x}|^{z}(z>1), \quad x \sim \hat{x}
$$

(for a normal quadratic extremum, $z=2$ ) for the same $z$ share identical metric properties. Thus, with $\lambda_{n} \rightarrow \lambda_{\infty}$, it is a consequence of this theory that

$$
\left|\lambda_{n}-\lambda_{\infty}\right| \propto \delta^{-n}
$$

with $\delta$ a function only of $z$. (For $z=2, \delta=4.6692016 \ldots$ ) That is, as $n \rightarrow \infty, \lambda_{n}$ converges to $\lambda_{\infty}$ at a universal geometric rate independent of the global properties of $f$.

By way of review, recall that a map possesses an $n$-cycle if there are $n$ points $x_{0}$, $x_{1}, \ldots, x_{n-1}$ such that

$$
x_{0} \stackrel{f}{\longrightarrow} x_{1} \stackrel{f}{\longrightarrow} \cdots \stackrel{f}{\longrightarrow} x_{r} \stackrel{f}{\longrightarrow} x_{r+1} \longrightarrow \cdots \stackrel{f}{\longrightarrow} x_{n-1} \stackrel{f}{\longrightarrow} x_{0} .
$$

Denoting the $n^{\text {th }}$ iterate of $f$ by $f^{n}$ :

$$
f^{n}(x)=f\left(f^{n-1}(x)\right) ; \quad f^{0}(x)=x
$$

where the $n$ elements of the cycle satisfy

$$
f^{n}\left(x_{r}\right)=x_{r} \quad r=0, \ldots, n-1
$$

i.e. each element is a fixed point of the $n^{\text {th }}$ iterate of $f$. Accordingly, the stability of a cycle is the stability of each element of the cycle viewed as a fixed point of $f^{n}$. If $x^{*}$ is a fixed point of $f$, stability is determined by linear approximation about $x^{*}$ : 
writing $x_{n}=x^{*}+\xi_{n}$,

$$
\begin{aligned}
\xi_{n+1} \cong & f^{\prime}\left(x^{*}\right) \xi_{n} \\
& \rightarrow \xi_{n} \cong \xi_{n}\left[f^{\prime}\left(x^{*}\right)\right]^{n} .
\end{aligned}
$$

$x^{*}$ is stable if $x_{n} \rightarrow x^{*}$ for $x_{0}$ sufficiently close to $x^{*}$. The criterion for stability is evidently

$$
\left|f^{\prime}\left(x^{*}\right)\right|<1
$$

while $f^{\prime}\left(x^{*}\right)=0$ is the condition for greatest stability. For an $n$-cycle the condition for stability is then

$$
\left|f^{n^{\prime}}\left(x_{r}\right)\right|<1 \text { for each } r .
$$

Indeed, by the chain rule, $f^{n^{\prime}}$ is independent of $r$ with

$$
f^{n^{\prime}}\left(x_{r}\right)=f^{\prime}\left(x_{0}\right) f^{\prime}\left(x_{1}\right) \cdots f^{\prime}\left(x_{n-1}\right) .
$$

Accordingly, a most stable $n$-cycle is one containing the point $\hat{x}$, and the parameter value $\lambda_{n}$ determined as a zero of

$$
F_{n}\left(\lambda_{n}\right) \equiv f^{2^{n}}\left(\lambda_{n}, \hat{x}\right)-\hat{x} .
$$

Evidently $\lambda_{r}, r=0,1, \ldots, n-1$ are also zeroes of (20) together with many other values. Thus, $\lambda_{n}$ is defined recursively: $\lambda_{0}$ is in general unique; $\lambda_{1}$ is a different zero of $F_{1}$ closest to $\lambda_{0}$, etc. with $\lambda_{n}$ monotone in $n$. As $n$ becomes large, $F_{n}$ is increasingly time-consuming to compute while its zeroes become arbitrarily close. For large $n$, it is essentially impossible to locate $\lambda_{n}$ without knowledge of (19), which is used as

$$
\lambda_{n+2}-\lambda_{n+1} \cong \delta^{-1}\left(\lambda_{n+1}-\lambda_{n}\right)
$$

to predict the next value given two previous values. (Indeed, a host of lengthy numerical studies have, in the past, observed the pattern 1, 2, aperiodic simply because $\lambda_{\infty}-\lambda_{1} \sim \lambda_{1}-\lambda_{0}$, while equal-parameter increment searches were performed.)

The theory actually determines (19) (and $\delta$ ) secondarily, with a scaling phenomenon on the elements of a cycle primary. At $\lambda_{n}$ a maximally stable $2^{n}$ cycle exists. As $\lambda$ is increased through some interval, a stable $2^{n}$-cycle persists until at $\Lambda_{n}$ it loses stability "bifurcating" into a $2^{n+1}$ cycle. For $\lambda$ slightly above $\Lambda_{n}, 2^{n}$ iterations map $x_{0}$ into a point arbitrarily near to $x_{0}$ for $\lambda$ arbitrarily near to $\Lambda_{n}$. Indeed, throughout the interval of $\lambda$ up to $\Lambda_{n+1}, f^{2}\left(x_{r}\right)$ is that element of the $2^{n+1}$ cycle nearest to but distinct from $x_{r}$, and

$$
\psi_{r}^{(n+1)} \equiv x_{r}-f^{2^{n}}\left(x_{r}\right)\left(\Lambda_{n}<\lambda<\Lambda_{n+1}\right)
$$

is a measure of deviation from $2^{n}$-cycle behavior throughout the cycle. [The reader will of course realize that (22) is simply the discrete, one-dimensional version of (2).] The basic result of the theory is that

$$
\hat{x}-f^{2^{n}}\left(\lambda_{n+1}, \hat{x}\right) \cong-\alpha^{-1}\left(\hat{x}-f^{2^{n-1}}\left(\lambda_{n}, \hat{x}\right)\right)
$$


[compare with Eq. (4)] where $\alpha$ enjoys a universality identical to $\delta$ 's, with value (for $z=2$ )

$$
\alpha=2.502907875 \ldots
$$

Moreover, in units of the distance between $\hat{x}$ and $f^{2^{n-1}}\left(\lambda_{n}, \hat{x}\right)$ the locations of the elements of the $2^{n}$-cycle about $\hat{x}$ are universally determined through a function $g_{0}(x)$ where theory determines that

$$
\lim _{n \rightarrow \infty}(-\alpha)^{n}\left[f^{2^{n}}\left(\lambda_{n}, \hat{x}+\xi /(-\alpha)^{n}\right)-\hat{x}\right]=v g_{0}(\xi / v)
$$

with the magnification $v$ the only $f$-dependent ingredient. [Upon remagnification, so that $\hat{x}=f^{2^{n-1}}\left(\lambda_{n}, \hat{x}\right) \rightarrow 1$ (i.e. setting the scale as above), a universal limit is obtained.] The elements of the $2^{n}$ cycle have the property of being fixed points of $g_{0}$ at its extrema, while $g_{0}$ itself can be universally computed by the theory. Related to $g_{0}$ is a sequence of universal functions $g_{r}$ where

$$
\lim _{n \rightarrow \infty}(-\alpha)^{n}\left[f^{2^{n}}\left(\lambda_{n+r}, \hat{x}+\xi /(-\alpha)^{n}\right)-\hat{x}\right]=v g_{r}(\xi / v)
$$

with the same $v$ as in (24). [The universal scale is simply $g_{1}(0)=1$.] These functions serve as a basis as which the operator of functional composition and rescaling becomes the shift:

$$
g_{r-1}(x)=-\alpha g_{r}\left(g_{r}(-x / \alpha)\right) \equiv O\left[g_{r}\right] .
$$

Since $g_{r}$ is universal, and since for an $f$ symmetric about $\hat{x}$, the limit in (25) is a function symmetric in $\xi$, each $g_{r}$ is a symmetric function, so that (26) can be written as

$$
g_{r-1}(x)=-\alpha g_{r}\left(g_{r}(x / \alpha)\right) .
$$

In particular

$$
g(x) \equiv \lim _{r \rightarrow \infty} g_{r}(x)
$$

is the fixed-point of $O$ :

$$
g(x)=-\alpha g(g(x(\alpha))) .
$$

(27) admits of a unique solution for $\alpha$ and $g(x)$ for $g(x)$ with $z^{\text {th }}$ order extremum at 0 , symmetric, and of sufficient smoothness. $g_{r}$ for large $r$ is obtained by linearizing $O$ about $g$ and studying the spectrum. Indeed the linearized operator has a unique eigenvalue in excess of one, this eigenvalue $\delta$ of (19). (References [13,14] maintained the uniqueness of $\delta$ as a necessary conjecture for the validity of the entirety of this theory. Recently this conjecture has been rigorously proven $[16,17]$, although only for $z$ sufficiently close to one.)

The verbal content of $(23)$ is that points near $\hat{x}$ scale in successive bifurcations by $-\alpha$. Since these points are imaged by $f$ to a right-most cluster of points (taking the extremum to be a maximum), and $f$ has a $z^{\text {th }}$ order extremum, it follows that points about $f(\hat{x})$ must scale by $\alpha^{z}$, i.e.

$$
f\left(\lambda_{n+1}, \hat{x}\right)-f^{2^{n}}\left(\lambda_{n+1}, f\left(\lambda_{n+1}, \hat{x}\right)\right) \cong \alpha^{-z}\left(f\left(\lambda_{n}, \hat{x}\right)-f^{2^{n-1}}\left(\lambda_{n}, f\left(\lambda_{n}, \hat{x}\right)\right)\right) .
$$


The next several images of this cluster of points are through a slope of an infinitesimal linear stretch of $f$, and so also scale with $\alpha^{z}$. Similarly, several pre images of the cluster about $\hat{x}$ scale with $-\alpha$ (several here means $r$, with $\frac{1}{n} \log r \ll 1$ for a $2^{n}$-cycle). Our immediately task is to determine this scale factor along the entirety of the cycle. Towards this end write

$$
\psi_{r}^{(n+1)} \equiv \sigma_{r}^{(n+1)} \psi_{r}^{(n)}
$$

setting $x_{0} \equiv \hat{x}$. By $(23) \sigma_{0}^{(n)}=-\alpha^{-1}, \sigma_{1}^{(n)}=\alpha^{-z}$. Also, $\sigma_{1}^{(n)}=\sigma_{2}^{(n)}=\ldots$ and $\sigma_{0}^{(n)}=\sigma_{-1}^{(n)}$ $=\sigma_{-2}^{(n)}=\ldots$, as $n \rightarrow \infty$. By the definitions of $\psi$ in Eq. (22)

$$
\begin{aligned}
& \psi_{2^{n+r}}^{(n+1)}=f^{2^{n}}\left(\lambda_{n+1}, x_{r}\right)-f^{2^{n}}\left(\lambda_{n+1}, f^{2^{n}}\left(\lambda_{n+1}, x_{r}\right)\right) \\
& =f^{2^{n}}\left(\lambda_{n+1}, x_{r}\right)-f^{2^{n+1}}\left(\lambda_{n+1}, x_{r}\right) \\
& =f^{2 n}\left(\lambda_{n+1}, x_{r}\right)-x_{r}
\end{aligned}
$$

or,

$$
\psi_{2^{n}+r}^{(n+1)}=-\psi_{r}^{(n+1)}
$$

while

$$
\psi_{2^{n+1}+r}^{(n+1)}=\psi_{r}^{(n+1)} .
$$

Thus,

$$
\sigma_{2^{n+r}}^{(n+1)}=-\sigma_{r}^{(n+1)}
$$

and in particular,

$$
\sigma_{2^{n}}^{(n+1)}=\alpha^{-1} .
$$

It is clear that deviations from $-\alpha^{-1}, \alpha^{-z}$ can occur only for $r$ such that

$$
\lim _{n \rightarrow \infty} r / 2^{n} \neq 0 .
$$

Accordingly, choose $r$ as

$$
r_{m} \equiv 2^{n-m}
$$

so that

$$
\begin{aligned}
\psi_{r_{m}}^{(n)} & =f^{2^{n-m}}\left(\lambda_{n}, \hat{x}\right)-f^{2^{n-1}}\left(\lambda_{n}, f^{2^{n-m}}\left(\lambda_{n}, \hat{x}\right)\right) \\
& =f^{2^{n-m}}\left(\lambda_{n}, \hat{x}\right)-f^{2^{n-m}}\left(\lambda_{n}, f^{2^{n-1}}\left(\lambda_{n}, \hat{x}\right)\right) .
\end{aligned}
$$

By $(25), f^{2^{n-1}}\left(\lambda_{n}, \hat{x}\right) \sim \frac{v}{(-\alpha)^{n-1}} g_{1}(0)+\hat{x}$ so that, again by $(25)$,

$$
\begin{aligned}
f^{2^{n-m}}\left(\lambda_{n}, f^{2^{n-1}}\left(\lambda_{n}, \hat{x}\right)\right) & \sim \frac{v}{(-\alpha)^{n-m}} g_{m}\left[\frac{(-\alpha)^{n-m}}{v} \frac{v}{(-\alpha)^{n-1}} g_{1}(0)\right]+\hat{x} \\
& =\frac{v}{(-\alpha)^{n-m}} g_{m}\left(\alpha^{1-m} g_{1}(0)\right)+\hat{x} .
\end{aligned}
$$


Similarly,

$$
f^{2^{n-m}}\left(\lambda_{n}, \hat{x}\right) \sim \frac{v}{(-\alpha)^{n-m}} g_{m}(0)+\hat{x}
$$

so that

$$
\psi_{r_{m}}^{(n)} \sim \frac{v}{(-\alpha)^{n-m}}\left[g_{m}(0)-g_{m}\left(\alpha^{1-m} g_{1}(0)\right)\right] .
$$

At $n+1$, to maintain the same number of iterations into the cycle, $r_{m}$ must be unchanged, so that by (33) $m \rightarrow m+1$, and (35) becomes

$$
\psi_{m+1}^{(n+1)} \sim \frac{v}{(-\alpha)^{n-m}}\left[g_{m+1}(0)-g_{m+1}\left(\alpha^{-m} g_{1}(0)\right)\right] .
$$

Finally, by (29)

$$
\sigma_{r_{m}}^{(n+1)} \sim \frac{g_{m+1}(0)-g_{m+1}\left(\alpha^{-m} g_{1}(0)\right)}{g_{m}(0)-g_{m}\left(\alpha^{1-m} g_{1}(0)\right)}
$$

which as $n \rightarrow \infty$ is exact. Defining $x_{m} \equiv r_{m} / 2^{n+1}=2^{-m-1}, \sigma\left(x_{m}\right) \equiv \lim _{n \rightarrow \infty} \sigma_{r_{m}}^{(n)}$

$$
\sigma\left(2^{-m-1}\right)=\frac{g_{m+1}(0)-g_{m+1}\left(\alpha^{-m} g_{1}(0)\right)}{g_{m}(0)-g_{m}\left(\alpha^{1-m} g_{1}(0)\right)} .
$$

$\left(\sigma_{r}^{(n)}\right.$ is defined on integers $r$; we have rescaled $r$ by $2^{n}$ so that an entire cycle, for any $n$ occupies $x \in[0,1]$. By (31) $\sigma(x)$ obeys (5) for, as $n \rightarrow \infty$, all $x \in[0,1]$.) Since the $g_{m}$ are all universal, it now follows that $\sigma\left(2^{-m}\right)$ is universal. For $m=0,(37)$ reads

$$
\sigma(1 / 2)=\frac{g_{1}(0)-g_{1}\left(g_{1}(0)\right)}{g_{0}(0)-g_{0}\left(\alpha g_{1}(0)\right)}
$$

But, by $(24), g_{0}(0)=0$, while by $(26)$,

$$
\begin{aligned}
0 & =g_{0}(0)=-\alpha g_{1}\left(g_{1}(0)\right) \quad g_{1}\left(g_{1}(0)\right)=0 \\
g_{0}\left(\alpha g_{1}(0)\right) & =-\alpha g_{1}\left(g_{1}\left(g_{1}(0)\right)\right)=-\alpha g_{1}(0)
\end{aligned}
$$

so that

$$
\sigma(1 / 2)=\alpha^{-1}
$$

which is obviously correct since $\sigma(1 / 2)=-\sigma\left(0^{-}\right)=-\left(-\alpha^{-1}\right)$. Also for $m \rightarrow \infty$

$$
\sigma\left(0^{+}\right)=\lim _{m \rightarrow \infty} \frac{g(0)-g\left(\alpha^{-m} g_{1}(0)\right)}{g(0)-g\left(\alpha^{1-m} g_{1}(0)\right)}
$$

But for $x$ small, $g(x)=g(0)+1 / 2 g^{\prime \prime}(0) x^{2}+\ldots$, so that $\sigma\left(0^{+}\right)=\alpha^{-2}$. However, $m=1$ is non-trivial and represents a drastic change in scaling half-way around the cycle:

$$
\sigma(1 / 4)=\frac{g_{2}(0)-g_{2}\left(g_{1}(0) / \alpha\right)}{g_{1}(0)-g_{1}\left(g_{1}(0)\right)} .
$$


This requires a computation using a numerically computed $g_{2}$, with result

$$
\sigma(1 / 4)=0.1752 \ldots
$$

We now compute $\psi_{r_{m+1}}^{(n)}$, which through interchanges of numbers of iterations yields

$$
\begin{aligned}
\psi_{r_{m+1}}^{(n)} & \sim f\left(\lambda_{n}, \hat{x}+\frac{v}{(-\alpha)^{n-m}} g_{m}(0)\right)-f\left(\lambda_{n}, \hat{x}+\frac{v}{(-\alpha)^{n-m}} g_{m}\left(\alpha^{1-m} g_{1}(0)\right)\right) \\
& \sim \frac{1}{2} f^{\prime \prime}\left(\lambda_{n}, \hat{x}\right) \frac{v^{2}}{\left(\alpha^{2}\right)^{n-m}}\left[g_{m}^{2}(0)-g_{m}^{2}\left(\alpha^{1-m} g_{1}(0)\right)\right]
\end{aligned}
$$

so that

$$
\sigma_{r_{m+1}}^{(n+1)} \sim \frac{g_{m+1}^{2}(0)-g_{m+1}^{2}\left(\alpha^{-m} g_{1}(0)\right)}{g_{m}^{2}(0)-g_{m}^{2}\left(\alpha^{1-m} g_{1}(0)\right)}
$$

which produces for $n \rightarrow \infty$

$$
\sigma\left(2^{-m-1}+0\right)=\frac{g_{m+1}^{2}(0)-g_{m+1}^{2}\left(\alpha^{-m} g_{1}(0)\right)}{g_{m}^{2}(0)-g_{m}^{2}\left(\alpha^{1-m} g_{1}(0)\right)} .
$$

Special cases of (38) are

$$
\sigma(1 / 2+0)=-\alpha^{-2}
$$

and $\sigma(1 / 4+0)=0.4191 \ldots$.

Thus, at $1 / 4$ an abrupt change from small to large scaling occurs : in essentially one iteration the string of images of the right most cluster suddenly goes over into the string of pre-images leading to $\hat{x}$.

So far, the largest discontinuity of $\sigma(x)$ has been computed, as well as successively smaller discontinuities at $x=2^{-m-1}$. It is now straight forward to compute the discontinuities at any rational $x$ given its binary representation. All that is necessary is to write

$$
r_{\{m\}}^{(n)}=2^{n-m_{1}}+2^{n-m_{2}}+\ldots
$$

and observe that

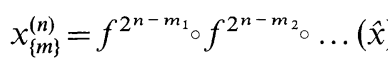

where $\circ$ denotes functional compositions. Repeated use of (25) then produces

$$
\begin{aligned}
& \sigma\left(2^{-m_{1}-1}+2^{-m_{2}-1}+\ldots\right) \\
& \quad=\frac{\left.\left[\ldots g_{m_{2}+1}\left(\alpha^{m_{1}-m_{2}} g_{m_{1}+1}(0)\right) \ldots\right)\right]-\left[\ldots g_{m_{2}+1}\left(\alpha^{m_{1}-m_{2}} g_{m_{1}+1}\left(\alpha^{-m_{1}} g_{1}(0)\right) \mid \ldots\right)\right]}{\left.\left.\left[\ldots g_{2}\left(\alpha_{m}^{m_{1}-m_{2}} g_{1}(0)\right)_{m} \ldots\right)\right]-\left[\ldots g_{2}\left(\alpha_{m}^{m_{1}-m_{2}} g_{1}\left(\alpha_{m}^{1-m_{1}} g(0)\right)\right)_{1} \ldots\right)\right]}
\end{aligned}
$$

for the value of $\sigma$ to the left of its discontinuity at $x$, while $\sigma$ to the right of the discontinuity is simply (39) with each bracketed term squared. (These "composite" discontinuities rapidly decrease in amplitude.) Accordingly, $\sigma(x)$ is discontinuous on the rationals with successively milder strength in successive half fractions of intervals, but universal. Figure 2 depicts the universally computed $\sigma(x)$ which upon 
comparison with Fig. 1 is evidently the $n \rightarrow \infty$ limit of the differential equation's $\sigma$. Indeed the quoted values for $\mu$, etc. are precisely the values of the universal $\sigma$ computed on the previous few pages. At this point it is computer-experimentally plausible that $\sigma(x)$ is universal over systems (1) - Duffings's equation was drawn at random as an example. Indeed we are asserting that $\sigma$ arises in Lorenz' system, in a five mode truncation of Navier-Stokes on the torus, the "three wave" problem and probably, based on the spectral test for the Bénard flow experiment, in the Navier-Stokes field equations with whatever corrections to these equations that are required to determine physical Bénard flow!

It now remains to establish why the one-dimensional universality theory presented in this section should apply to these higher dimensional flows.

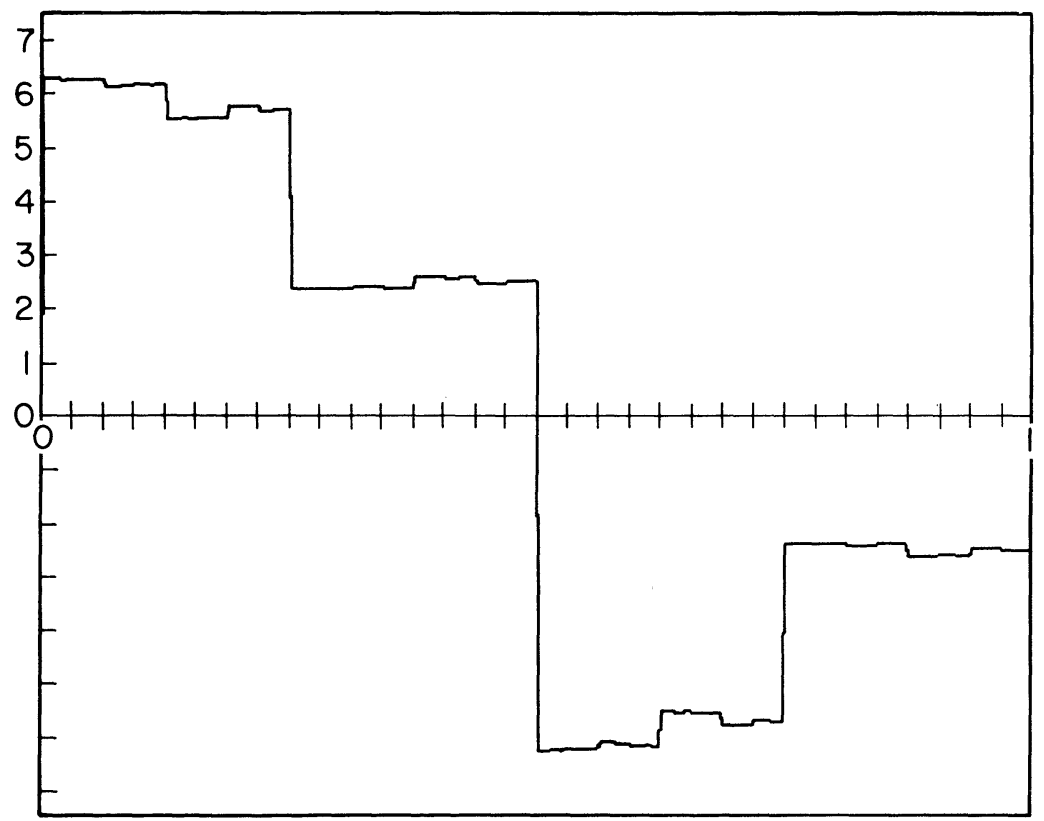

Fig. 2. $\sigma^{-1}$ as universally computed

\section{The Extension of Universality to High Dimensional Flows}

Our first task is to extend the previous section to $N$ dimensional maps [18]. Next the connection between these maps and the flow of a system of differential equations is to be drawn. Finally several details will be fixed.

The first task has been performed by Collet et al. [9]. The basic result is that if a one parameter family of maps (after a coordinate transformation if necessary) passes near to the map

$$
\left(\begin{array}{c}
x_{1} \\
x_{2} \\
\vdots \\
x_{N}
\end{array}\right) \rightarrow\left(\begin{array}{c}
g\left(\left(x_{1}^{2}-x_{2}-\ldots-x_{N}\right)^{1 / 2}\right) \\
0 \\
\vdots \\
0
\end{array}\right),
$$


then it will go through $2^{n}$ bifurcations ad infinitum, and exhibit essentially one dimensional behavior. That is, about a given element of a cycle, the iterated map collapses about a definite (local) ray in the $\mathrm{N}$-dimensional space, and along this ray the mapping is one-dimensional and obeys the universality theory of the previous section. In contrast to the one-dimensional case, it is difficult in $N$ dimensions to know if a map will be of this nature by a casual perusal of its explicit form. Numerically, however; a large variety of interesting systems exhibit this behavior.

We next turn to the connection between maps and flows. The basic idea here is the "Poincare map". The solution to (1) is a trajectory in $N$ dimensions which, for motions of interest here, lies in a compact region performing a "noisy" periodic motion. A map is defined by slicing transversally across the trajectory with an $N-1$ dimensional plane: each time the orbit crosses this plane in the same sense (e.g. from "below") mark its $N-1$ coordinates. The image of one such point in the plane is by definition the location of its next crossing. This map is called the Poincaré map and by elementary properties of (1) is invertible and differentiable. Should the orbit close on itself after one intersection with the plane, then the map has a fixed point; should it close after $n$ crossings (or "basic cycles") then the map has an $n$-cycle. Finally, should it never close, then the map has no periodic cycle. Also, should an $n$-cycle of the map be stable, then by uniqueness and smoothness of the solution to (1), the orbit will converge to a definite stable orbit with identical convergence around the entire orbit. Accordingly all stability and periodicity aspects of the trajectory can be deduced from the map. It now follows by the previous paragraph that the universality theory can apply to (1). To be precise, for an appropriate $\lambda$, the trajectory returns every $2^{p}$ times successively closer to an original point along a certain ray in the $N-1$ dimensional plane. At $\lambda_{n}$, after $2^{n-1}$ crossings the trajectory has returned to the nearest adjacent point to its original crossings, and the points at $2^{n}, 2^{n-1}, 2^{n-2}, \ldots, 2^{n-m} m \ll n$ all lie along this special ray at spacings determined by $g_{0}$. By linearity, the same is true for any one-dimensional projection of the $N-1$ dimensional plane. Accordingly, we immediately have (19) satisfied for the parameter, and in each projection, a scaling of the form (23) from one bifurcation to the next. Now, consider two adjacent points $y, z$ in the $N-1$ plane such that their spacing scales by $\sigma_{0}$. After one iteration of the map, they are adjacent points $y^{\prime}, z^{\prime}$ also scaling by $\sigma_{0}$ [i.e. they are not located at a large discontinuity of $\sigma(x)]$. Issuing out of $y$ is a trajectory "cycle" linking it to $y^{\prime}$; adjacent to this "cycle" is the trajectory linking $z$ to $z^{\prime}$. It now follows by the differential flow that these two trajectories have a spacing that also scales with $\sigma_{0}$ along its entire length from $y$ to $y^{\prime}$. Now the $z$ adjacent to $y$ is $f^{2^{n-1}}(y)$. But $2^{n-1}$ crossings later require a time $2^{n-1} T_{0} \equiv T_{n-1}$ where $T_{0}$ is the time of a "basic cycle". Accordingly, $\psi^{(n)}(t)$ of Eq. (2) is precisely the spacing of the two adjacent trajectories linking $y, y^{\prime}$ and $z, z^{\prime}$. From (29) we now immediately conclude (4) where $\sigma(x)$ is the universal scaling function of the previous section. Accordingly, if (1) has successive half-subharmonic behavior, then the spectrum of each projection $x_{i}(t)$ is determined by (16) with the universal function $\sigma$. [The map restricted to the special ray must generically be of quadratic sort, since it is differentiable, and $z \neq 2$ implies that $f^{\prime \prime}(\hat{x})=0$ which is non-generic.] These remarks, then, establish the general applicability of the one-dimensional theory. 
Let us comment on some mathematical features of $\sigma$. Recall that

$$
\frac{\psi^{(n+1)}(t)}{\psi^{(n)}(t)} \sim \sigma\left(t / T_{n+1}\right)
$$

Denoting $x^{(n)}(0) \equiv \hat{x}_{n}, x^{(n)}\left(m T_{0}\right)=P^{m}(\hat{x})$ where $P$ is the Poincare map at $\lambda_{n}$, so that

$$
\psi^{(n+1)}\left(m T_{0}\right)=P^{m}\left(\hat{x}_{n+1}\right)-P^{m} \circ P^{2^{n}}\left(\hat{x}_{n+1}\right) .
$$

( $P$ is always understood to be the map at the $\lambda$ value corresponding to the subscript of $\hat{x}$.) Thus,

$$
\sigma\left(m T_{0} / T_{n+1}\right)=\sigma\left(m / 2_{n+1}\right) \cong \frac{P^{m}\left(\hat{x}_{n+1}\right)-P^{m} \circ P^{2^{n}}\left(\hat{x}_{n+1}\right)}{P^{m}\left(\hat{x}_{n}\right)-P^{m} \circ P^{2^{n-1}}\left(\hat{x}_{n}\right)} .
$$

That is, $\sigma$ is determined at the $n+1^{\text {st }}$ level of bifurcation at the rationals whose denominator is $2^{n+1}$ purely by the Poincaré map, and as $n \rightarrow \infty$ at the rationals generally purely by the Poincaré map with $\sigma$ determined at all reals by its continuous extension. Moreover, at $m=2^{n-r}+2^{n-s}+\ldots$ for a finite such sum and $r, s \ldots \ll n$, as $n \rightarrow \infty \sigma$ converges to the universality values (and discontinuities) of (39). Also, for $m=2^{n-r}+2^{n-s}+\ldots+p$ for $p=1,2, \ldots$ while $\frac{1}{n} \ln p \ll 1, \sigma$ is independent of $p$. Thus as $n \rightarrow \infty$ the continuous extension of $\sigma$ has the property of $\sigma^{\prime}(x)=0$ almost everywhere with discontinuities at the rationals such that if $\sigma$ has a discontinuity $d$ at a rational $r$, then for rationals arbitrarily close to $r$, the associated discontinuities are arbitrarily smaller than $d$.

It is important to point out that not all scaling laws deduced from the associated map can extend to the trajectories. For example it is a direct consequence of the meaning of $\alpha$ that

$$
\lim _{n \rightarrow \infty} \frac{P^{2 m}\left(\hat{x}_{n+1}\right)-P^{2 m}\left(\hat{x}_{n}\right)}{P^{m}\left(\hat{x}_{n}\right)-P^{m}\left(\hat{x}_{n-1}\right)}=\alpha^{-1} \text { for all } m .
$$

However, the analogous trajectory formula

$$
\frac{x^{(n+1)}(2 t)-x^{(n)}(2 t)}{x^{(n)}(t)-x^{(n-1)}(t)} \rightarrow \alpha^{-1} \text { for all } t
$$

is necessarily false. Rather, it is correct for $t_{m}=m T_{0}$, but widely oscillates over the duration of a basic cycle. Only map scaling-laws pertaining to the same time can be lifted by the flow to become trajectory laws. For example, let us formally deduce this property for $\sigma$. By (1),

$$
\dot{x}^{(n+1)}(t)=f\left(x^{(n+1)}(t), \lambda_{n+1}\right)
$$

and

$$
\dot{x}^{(n+1)}\left(t+T_{n}\right)=f\left(x^{(n+1)}\left(t+T_{n}\right), \lambda_{n+1}\right),
$$

so that

$$
\dot{\psi}^{(n+1)} \cong D f\left(x^{(n+1)}(t), \lambda_{n+1}\right) \cdot \psi^{(n+1)} \equiv M^{(n+1)}(t) \cdot \psi^{(n+1)} .
$$


By definition,

$$
\psi^{(n+1)}=\sigma_{n}(t) \psi^{(n)} \quad\left(\sigma_{n} \text { is a scalar }\right) .
$$

Differentiating (41) and utilizing (40) and the analogous formula for $n+1 \rightarrow n$,

$$
M^{(n+1)} \cdot \psi^{(n+1)}=\sigma_{n} M^{(n+1)} \cdot \psi^{(n)}=\dot{\sigma}_{n} \psi^{(n)}+\sigma_{n} \dot{\psi}^{(n)}=\dot{\sigma}_{n} \psi^{(n)}+\sigma_{n} M^{(n)} \cdot \psi^{(n)}
$$

or,

$$
\dot{\sigma}_{n} \psi^{(n)} \cong \sigma_{n}\left(M^{(n+1)}-M^{(n)}\right) \cdot \psi^{(n)} .
$$

However, $\lambda_{n+1}-\lambda_{n} \sim \delta^{-n}$ and also $x^{(n+1)}(t)-x^{(n)}(t) \sim \delta^{-n}$, so that

$$
\dot{\sigma}_{n} \psi^{(n)} \sim \delta^{-n} \sigma_{n} N(t) \cdot \psi^{(n)}
$$

for an appropriate $N(t)$. Accordingly, as $n \rightarrow \infty, \dot{\sigma}_{n} \rightarrow 0$ for $\psi^{(n)}(t) \neq 0$. Thus, $\sigma$ is preserved at the value set by the map, readjusting to its new values at those points during the basic cycle at which $\psi^{(n)}$ vanishes. (These are, of course, the discontinuities of $\sigma$ occurring precisely where the ratio $\sigma$ is undefined.) $\mathrm{Had} \psi^{(n)}$ and $\psi^{(n+1)}$ been evaluated at different times - in particular at a ratio of two - then the right hand side of (42) would be oscillating at a scale of $x(t)$ and the corresponding ratio could not remain constant. It is worthy to mention at this point that the trajectory constancy of $\sigma$ implies that the basic spectral recursion (16) holds for all spectral components, and not just below $\omega_{0}$ - the purely subharmonic components would still obey (16) had $\sigma$ merely averaged to the map value over each basic cycle, while the harmonics of these components at and above the basic frequency would fail to do so. Thus, the way in which the basic cycle itself metamorphoses as $n \rightarrow \infty$ is also determined by the theory.

We now turn to the recursion for the even spectral components. By (9)

$$
x_{2 k}^{(n+1)}-x_{k}^{(n)}=\int_{0}^{T_{n}} \frac{d t}{2 T_{n}}\left[x^{(n+1)}(t)+x^{(n+1)}(t+T)-2 x^{(n)}(t)\right] e^{-2 \pi i k t / T_{n}}
$$

or,

$$
\begin{aligned}
x_{2 k}^{(n+1)}-x_{k}^{(n)}= & \int_{0}^{T_{n}} \frac{d t}{T_{n}}\left[x^{(n+1)}(t)-x^{(n)}(t)\right] e^{-2 \pi i k t / T_{n}} \\
& -\int_{0}^{T_{n}} \frac{d t}{2 T_{n}}\left[x^{(n+1)}(t)-x^{(n+1)}\left(t+T_{n}\right)\right] e^{-2 \pi i k t / T_{n}} .
\end{aligned}
$$

The second term of (43) is, of course, determined through $\sigma$, so that the new ingredient necessary to obtain a recursion is the function

$$
\frac{x^{(n+1)}(t)-x^{(n)}(t)}{x^{(n)}(t)-x^{(n-1)}(t)} \cong \varrho\left(t / T_{n+1}\right) .
$$

Formula (44) anticipates that the ratio of the left hand side has a dependence purely through the scale for $t$ (as $n \rightarrow \infty$ ) and, as the reader has no doubt guessed, has a universal limit. Now, for that $t$ one cycle, $T_{0}$, after $x^{(n)}=\hat{x}_{n},(19)$ implies that $\varrho=\delta^{-1}$; also $\varrho$ will persist at $\delta^{-1}$ for some range of $t$ above this value, lending 
evidence to the correctness of (44). To go further, consider once more

$$
t_{r}=2^{n-r} T_{0}
$$

so that

$$
\varrho\left(2^{-1-r}\right) \cong \frac{P^{2^{n-r}}\left(\hat{x}_{n+1}\right)-P^{2^{n-r}}\left(\hat{x}_{n}\right)}{P^{2^{n-r}}\left(\hat{x}_{n}\right)-P^{2^{n-r}}\left(\hat{x}_{n-1}\right)} .
$$

Again, employ the universality formulae (25) to obtain

$$
\varrho\left(2^{-1-r}\right) \cong \frac{\left(\hat{x}_{n+1}-\hat{x}_{n}\right)+\frac{v}{(-\alpha)^{n-r}}\left(g_{r+1}(0)-g_{r}(0)\right)}{\left(\hat{x}_{n}-\hat{x}_{n-1}\right)+\frac{v}{(-\alpha)^{n-r}}\left(g_{r}(0)-g_{r-1}(0)\right)} .
$$

Now the value $\hat{x}$ of the "extremal" point of the map is determined by some condition analogous to

$$
D_{x} f\left(\lambda_{n}, \hat{x}_{n}\right)=0
$$

in the 1-dimensional case. It thus follows from (19) that

$$
\hat{x}_{n+1}-\hat{x}_{n} \sim \eta \delta^{-n}
$$

for some $\eta$. Thus, (45) becomes

$$
\varrho\left(2^{-1-r}\right) \cong \frac{\eta / v\left(\frac{-\alpha}{\delta}\right)_{n}+(-\alpha)^{r}\left(g_{r+1}(0)-g_{r}(0)\right)}{\delta \eta / v\left(\frac{-\alpha}{\delta}\right)^{n}+(-\alpha)^{r}\left(g_{r}(0)-g_{r-1}(0)\right)} .
$$

Since $\alpha=2.5, \ldots, \delta=4.6, \ldots, \alpha / \delta<1$ and so, as $n \rightarrow \infty$,

$$
\varrho\left(2^{-1-r}\right)=\frac{g_{r+1}(0)-g_{r}(0)}{g_{r}(0)-g_{r-1}(0)}
$$

By an analysis identical to that that led to (39), it is clear that $\varrho$ is again universally determined at the rationals. However, should we attempt the deduction that led to the discontinuities of $\sigma$ [e.g. Eq. (38) derivation], the $\delta^{-n}$ term remains in numerator and denominator, while instead of $\alpha^{-n}$, we now have $\alpha^{-2 n}$ :

$$
\begin{aligned}
P \cdot P^{2^{n-r}}\left(\hat{x}_{n+1}\right)-P \cdot P^{2^{n-r}}\left(\hat{x}_{n}\right) & \sim P\left(\hat{x}_{n+1}+\frac{v}{(-\alpha)^{n-r}} g_{n+1}(0)\right) \\
& -P\left(\hat{x}_{n}+\frac{v}{(-\alpha)^{n-r}} g_{r}(0)\right) \\
\cong & \eta^{\prime} \delta^{-n}+\frac{1}{2} P^{\prime \prime}(\hat{x}) \frac{v^{2}}{\alpha^{2(n-r)}}\left(g_{r+1}^{2}(0)-g_{r}^{2}(0)\right) .
\end{aligned}
$$

Since $\alpha^{2}>\delta$, we now have

$$
\varrho\left(2^{-1-r}+0\right)=\delta^{-1} \text {. }
$$


Thus, the discontinuities at the rationals are now determined purely by @'s deviations from $\delta^{-1}$, which are vanishing as $r \rightarrow \infty$, growing as $x$ departs from 0 . [As $r \rightarrow \infty,(46)$ is simply the convergence rate of $g_{r}(0)$ which is $\delta^{-1}$.] On the other hand, for several iterations after this first quadratic imagining, only a slope is involved, so that $\varrho$ remains at $\delta^{-1}$. Similarly several iterates prior to $2^{-1-r}, \varrho$ remains at $\varrho\left(2^{-1-r}-0\right)$. Next, when we consider the second generation rationals $2^{-1-r}+2^{-1-s}$, so that

$$
P^{2^{n-s}} P^{2^{n-r}}\left(\hat{x}_{n}\right) \cong P^{2^{n-s}}\left(\hat{x}_{n}+\frac{v}{(-\alpha)^{n-r}} g_{r}(0)\right) \cong \hat{x}_{n}+\frac{v}{(-\alpha)^{n-s}} g_{s}\left((-\alpha)^{r-s} g_{r}(0)\right)
$$

then for $s \gtrsim n / 2$ the $g$ terms again dominate as $n \rightarrow \infty$, so that

$$
\varrho\left(2^{-r-1}+2^{-s-1}\right)=\frac{g_{s+1}\left(\alpha^{r-s} g_{r+1}(0)\right)-g_{s}\left(\alpha^{r-s} g_{r}(0)\right)}{g_{s}\left(\alpha^{r-s} g_{r}(0)\right)-g_{s-1}\left(\alpha^{r-s} g_{r-1}(0)\right)}
$$

and the discontinuity is now correctly given by the same formula with each term replaced by its square. Thus, at all rationals arbitrarily close to those of large discontinuity, small discontinuities reign. However, $\varrho$ for these large values of $s$ is by the formula

$$
g_{s}(x) \sim g(x)-\delta^{-s} h(x)
$$

( $h$ has quadratic extremum at $x=0$ and is universal) also just $\delta^{-1}$. Accordingly, $\varrho^{\prime}(x)=0$ almost everywhere, and up to $x=1 / 4$ is "fairly" close to $\delta^{-1}$ ' $\varrho$ can also be shown to have this "constancy" to $\delta^{-1}$ for $3 / 4<x<1$. Figure 3 depicts the $\varrho$ of $(44)$ for Duffings equations, while Fig. 4 depicts $\varrho$ as determined by the quadratic recursion as the interval

$$
x_{n+1}=a-x_{n}^{2}
$$

for a 256-cycle. (In these figures, $x=0$ has been displaced to $x=1 / 4$ in order to about the "well-behaved" quarters of $\varrho$ to form its first half-cycle.) As the cycle length is increased, convergence is of an oscillating sort as higher $r$ values with their discontinuities are exposed. Nevertheless, since $\varrho$ is used only as a functional (in an integral) with the large excursions occurring where the numerator and denominator are crossing zero, it is clear that for half the cycle, $\varrho=\delta^{-1}$ is a very good approximation. Accordingly, the phase of $x^{(n)}(t)$ in (43) must be set to be $3 / 4$ through the cycle in order to have $0<t<T_{n}$ as the "smooth" half cycle of $\varrho$.

We now return to (43) and substitute the fourier expansions of the $x(t)$ 's:

$$
\begin{aligned}
x_{2 k}^{(n+1)}-x_{k}^{(n)}= & \sum_{k^{\prime}} x_{k^{\prime}}^{(n)} \int_{0}^{T_{n}} \frac{d t}{T} \varrho\left(t / 2 T_{n}\right) e^{2 \pi i\left(k^{\prime}-k\right) t / T_{n}} \\
& -\sum_{k^{\prime}} x_{k^{\prime}}^{(n-1)} \int_{0}^{T_{n}} \frac{d t}{T_{n}} \varrho\left(t / 2 T_{n}\right) e^{2 \pi i\left(2 k^{\prime}-k\right) t / T_{n}} \\
& -\sum_{k^{\prime}} x_{2 k^{\prime}+1}^{(n)} \int_{0}^{T_{n}} \frac{d t}{T_{n}} \sigma\left(t / 2 T_{n}\right) e^{2 \pi i\left(2 k^{\prime}+1-k\right) t / T_{n}} \\
= & \sum_{k^{\prime}}\left(x_{2 k^{\prime}}^{(n)}-x_{k^{\prime}}^{(n-1)}\right) \int_{0}^{T_{n}} \frac{d t}{T_{n}} \varrho\left(t / 2 T_{n}\right) e^{2 \pi i\left(2 k^{\prime}-k\right) t / T_{n}} \\
& +\sum_{k^{\prime}} x_{2 k^{\prime}+1}^{(n)} \int_{0}^{T_{n}} \frac{d t}{T_{n}}\left[\varrho\left(t / 2 T_{n}\right)-\sigma\left(t / 2 T_{n}\right)\right] e^{2 \pi i\left(2 k^{\prime}+1-k\right) t / T_{n}}
\end{aligned}
$$




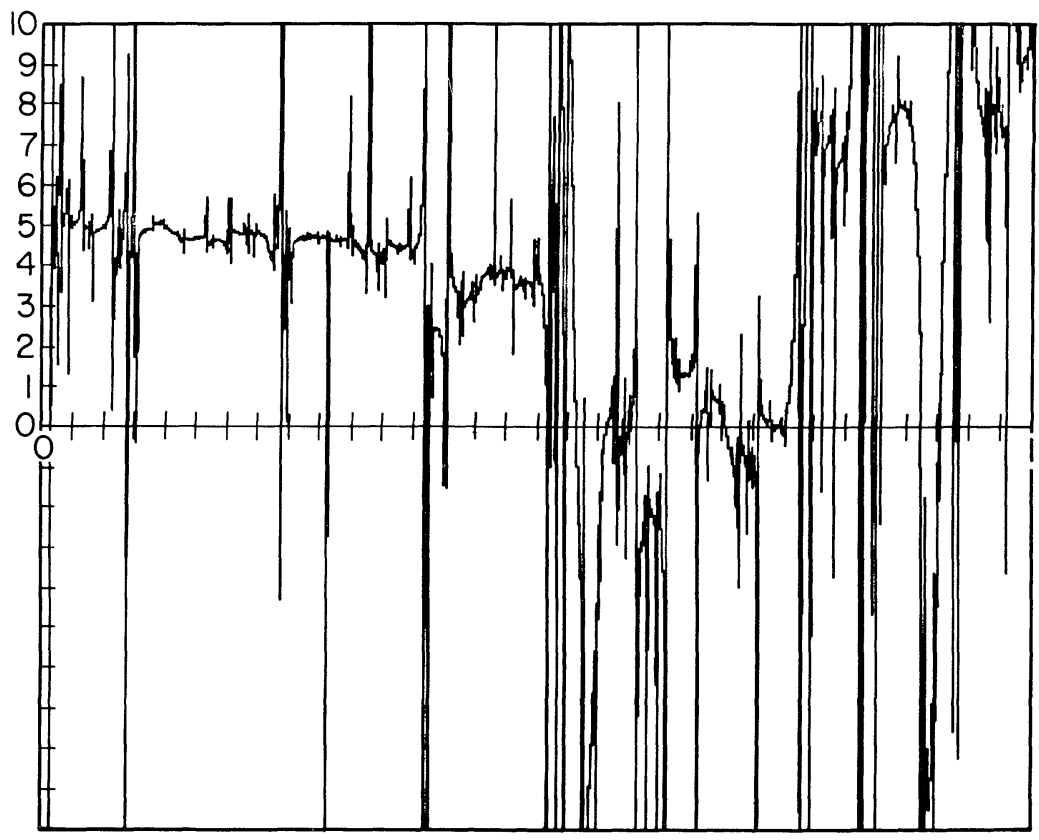

Fig. 3. $\varrho^{-1}$ of (44) for $n=4$ by numerical division of solutions to Duffing's equation

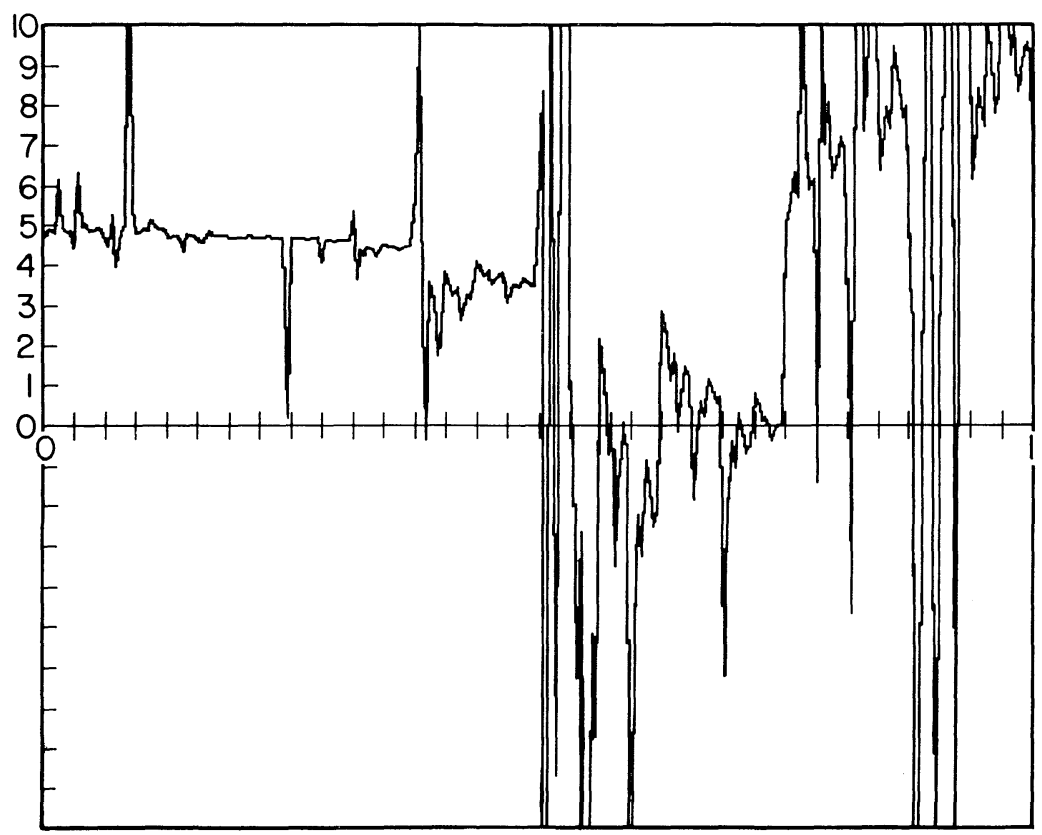


or,

$$
\begin{aligned}
x_{2 k}^{(n+1)}-x_{k}^{(n)}= & \sum\left[x_{2 k^{\prime}}^{(n)}-x_{k^{\prime}}^{(n-1)}\right] \int_{0}^{1} d \xi \varrho(\xi / 2) e^{2 \pi i \xi\left(2 k^{\prime}-k\right)} \\
& +\sum_{k^{\prime}} x_{2 k^{\prime}}^{(n)}+1 \int_{0}^{1} d \xi[\varrho(\xi / 2)-\sigma(\xi / 2)] e^{2 \pi i \xi\left(2 k^{\prime}+1-k\right)}
\end{aligned}
$$

It is immediately clear from (47) that the even spectrum is now compatible, and again through an autonomous universal recursion. However, the recurrence is now second order with superficially two levels of spectrum required to compute the next. Observe that the index in the first term (containing the second order term) is even in $k^{\prime}$, while odd in the second. With the $-1 / 4$ phasing, by the above discussion, $\varrho$ can be approximated by $\delta^{-1}$ in the first integral, so that

$$
\int_{0}^{1} d \xi \varrho(\xi / 2) e^{2 \pi i \xi\left(2 k^{\prime}-k\right)} \cong \delta^{-1} \delta_{2 k^{\prime}, k} .
$$

Thus to good approximation [since (47) is small to begin with] the recursion is second-order only for $k$ even. In particular, consider the "new" components, $x_{2 k+1}^{(n)}$, at the $n$th level: at the $n+1$ st level, the first term of (47) does not contribute, so that at the $n+1$ st level its value is determined by a first order recurrence:

$$
x_{2(2 k+1)}^{(n+1)}-x_{2 k+1}^{(n)} \cong \sum_{k^{\prime}} x_{2 k^{\prime}+1}^{(n)} \int_{0}^{1} d \xi[\varrho(\xi / 2)-\sigma(\xi / 2)] e^{4 \pi i\left(k^{\prime}-k\right)} .
$$

It is easy to obtain a good approximation to (49), recalling, though, that the $-1 / 4$ phasing determines $\sigma$ to be $-\alpha^{-1}$ on the first half of the integration, and $+\alpha^{-2}$ on the second:

$$
\begin{aligned}
\int_{0}^{1} d \xi[\varrho(\xi / 2)-\sigma(\xi / 2)] e^{4 \pi i \xi\left(k^{\prime}-k\right)} \cong & \delta^{-1} \delta_{k, k^{\prime}}+\alpha^{-1} \int_{0}^{1 / 2} d \xi e^{4 \pi i \xi\left(k^{\prime}-k\right)} \\
& -\alpha^{-2} \int_{0}^{1 / 2} d \xi e^{4 \pi i \xi\left(k^{\prime}-k\right)} \\
= & \left(\delta^{-1}+\frac{1}{2}\left(\alpha^{-1}-\alpha^{-2}\right)\right) \delta_{k^{\prime}, k} .
\end{aligned}
$$

Accordingly, we have the good approximation

$$
\begin{aligned}
x_{2(2 k+1)}^{(n+1)} & \cong\left(1+\delta^{-1}+\frac{1}{2}\left(\alpha^{-1}-\alpha^{-2}\right)\right) x_{2 k+1}^{(n)} \\
& =1.33 x_{2 k+1}^{(n)} .
\end{aligned}
$$

Thus, after a given component appears at some level, it maintains its phase and grows in amplitude by a universal factor of

$$
\mu^{\prime}=1.33
$$

or $10 \log _{10} \mu^{\prime} \cong 1.24 \mathrm{db}$.

Next consider even $k$, to determine the future growth of this component:

$$
\begin{aligned}
x_{4 k}^{(n+1)}-x_{2 k}^{(n)} \cong & \delta^{-1}\left[x_{2 k}^{(n)}-x_{k}^{(n-1)}\right] \\
& +\sum_{k^{\prime}} x_{2 k^{\prime}+1}^{(n)} \int_{0}^{1} d \xi\left[\delta^{-1}-\sigma(\xi / 2)\right] e^{2 \pi i \xi\left(2 k^{\prime}+1-2 k\right)} .
\end{aligned}
$$


But,

$$
\begin{aligned}
& \int_{0}^{1} d \xi\left[\delta^{-1}-\sigma(\xi / 2)\right] e^{2 \pi i \xi\left(2 k^{\prime}+1-2 k\right)}=-\int_{0}^{1} d \xi \sigma(\xi / 2) e^{2 \pi i \xi\left(2 k^{\prime}+1-2 k\right)} \\
& \cong \alpha^{-1} \int_{0}^{1 / 2} d \xi e^{2 \pi i \xi\left(2 k^{\prime}+1-2 k\right)}-\alpha^{-2} \int_{1 / 2}^{1} d \xi e^{2 \pi i \xi\left(2 k^{\prime}+1-2 k\right)} \\
& \quad=\left(\alpha^{-1}+\alpha^{-2}\right) \int_{0}^{1 / 2} d \xi e^{2 \pi i \xi\left(2 k^{\prime}+1-2 k\right)}=\frac{-\left(\alpha^{-1}+\alpha^{-2}\right)}{\pi i} \frac{1}{2 k^{\prime}+1-2 k}
\end{aligned}
$$

so that

$$
x_{4 k}^{(n+1)}-x_{2 k}^{(n)} \cong \delta^{-1}\left[x_{2 k}^{(n)}-x_{k}^{(n-1)}\right]-\frac{1}{\pi i}\left(\alpha^{-1}+\alpha^{-2}\right) \sum_{k^{\prime}} \frac{1}{2 k^{\prime}+1-2 k} x_{2 k^{\prime}+1}^{(n)} .
$$

To estimate the second term, use the principal value approximation to obtain

$$
+\frac{1}{2}\left(\alpha^{-1}+\alpha^{-2}\right) x_{n^{\prime \prime} k^{\prime \prime}}^{(n)} \cong \frac{1}{2}\left(\alpha^{-1}+\alpha^{-2}\right) x_{2 k+1}^{(n)} .
$$

$\left(x_{k^{\prime} k^{\prime \prime}}^{(n)}\right.$ is the interpolation of the odd $n$th order components, and so of magnitude of the closest of these.) But, by (18),

$$
x_{2 k+1}^{(n)} \sim 0.15 x_{2 k}^{(n)}
$$

when $k$ is odd, and smaller otherwise. Altogether, then, the second term of (52) is $\lesssim 0.04 x_{2 k}^{(n)}$, and so roughly negligible compared to the first term. Thus,

$$
x_{4 k}^{(n+1)}-x_{2 k}^{(n)} \cong \delta^{-1}\left[x_{2 k}^{(n)}-x_{k}^{(n-1)}\right] .
$$

Combined with (50) we now see that at the next levels the component under question grows first another $\sim 5 \%$, then $\sim 1 \%$ for a total further increase of several tenths of a decibel.

Altogether, we now summarize the construction of the spectrum. Start at the $n$th level, and smoothly interpolate the odd components. Shifted down by $8.2 \mathrm{db}$, this curve is the local average of the locations of the odd components of the $n+1$ st level. Next, shift up each of the odd components of the $n$th level by $1.4 \mathrm{db}$ to this new (and final) location. Finally, all the even components of the $n$th level remain in place. This (very good) approximation requires only the components at the $n$th level and now recursively determines the spectrum for $n \rightarrow \infty$. Whatever approximation is chosen, it is clear that the spectrum at the transition point is determined and our task is completed.

\section{Conclusions}

Our result is that given a system known to become erratic through a cascade of subharmonic bifurcations, after the spectrum is determined through some appropriate analysis through the first several bifurcations, the behavior down through the transition is determined. At the transition itself, the spectrum of any mode is comprised of $2^{n-1}$ components at the odd multiples of the $2^{n}$ subharmonic of the "original" frequency each of magnitude roughly $8.2 n \mathrm{db}$ below the basic component. The phases of these components are determined ultimately by the phases 
of the first bifurcations'components, although the basic formula (14) endows these high level components with rapid phase variations, so that they have a sort of random character. The total power in this spectrum (taken as the squares of the spectral amplitudes) is easily estimated at $\sim 2.5 \%$ of the power in the basic frequency, to give a rough idea of the transition noise.

Acknowledgements. The author has greatly profited from discussions with Jean-Pierre Eckmann, and accordingly thanks D. Bessis and NATO under whose auspices, at Cargèse, this contact occurred. A seminal discussion with my colleague Harvey Rose issued directly into the research of this paper. Finally, I am grateful to P. Carruthers, E. Lieb, and A. Jaffe for their enthusiastic reception of this work and facilitation of its rapid publication.

\section{References}

1. Franceschini, V., Tebaldi, C.: Sequences of infinite bifurcation and turbulence in five-modes truncation of the Navier-Stokes equations. Istituto Matematico, Univ. di Modena preprint

2. Franceschini, V.: A Feigenbaum sequence of bifurcations in the Lorenz model, Istituto Matematico, Univ. di Modena preprint, to be published in J. Stat. Phys.

3. Computations by the author on Duffing's equation, following Ueda, Y.: J. Stat. Phys. 20 (2), 181 (1979)

4. Holmes, P.: A nonlinear oscillator with a strange attractor. Department of Theoretical and Applied Mechanics, Cornell University (preprint)

5. Huberman, B., Crutchfield, J.: Chaotic states of anharmonic systems in periodic fields. Xerox Corp., Palo Alto Research Center (preprint)

6. Marzec, C.J., Spiegel, E.A.: A strange attractor. Astronomy Department, Columbia University (preprint)

7. Libchaber, A., Maurer, J.: Une expérience de Rayleigh-Bénard de géométrie réduite. École Normale Supérieure (preprint)

8. Feigenbaum, M.J.: Phys. Lett. 74A, 375 (1979)

9. Collet, P., Eckmann, J.-P., Koch, H.: Period doubling bifurcations for families of maps on $C^{n}$. Department of Physics, Harvard University (preprint)

10. Metropolis, N., Stein, M.L., Stein, P.R.: Combinatorial Theory 15 (1), 25 (1973)

11. May, R., Oster, G.: Amer. Naturalist 110 (974), 573 (1976). This paper independently of myself, discovers the first clue of a universal metric property

12. Feigenbaum, M.J.: Annual Report 1975-76, LA-6816-PR, Los Alamos

13. Feigenbaum, M.J.: J. Stat. Phys. 19 (1), 25 (1978)

14. Feigenbaum, M.J.: J. Stat. Phys. 21 (6) (1979)

15. Feigenbaum, M.J.: Lecture Notes in Physics 93, 163 (1979)

16. Collet, P., Eckmann, J.-P., Lanford III, O.: Universal properties of maps on an interval (in preparation)

17. Collet, P., Eckmann, J.-P.: Bifurcations et groupe de renormalisation. IHES/P/78/250 (preprint)

18. Derrida, B., Gervois, A., Pomeau, Y.: J. Phys. A 12, 269 (1979). This paper contains the first numerical observation of $\delta$ in a 2-dimensional map

Communicated by A. Jaffe

Received April 7, 1980; in revised form May 12, 1980 